\title{
Assessment of thyroid function in pregnant women attending Suez Canal University Hospitals
}

\begin{abstract}
Introduction: Pregnancy is a physiological state in which significant changes in thyroid function occur. Several factors contribute to these changes. These factors could contribute to thyroid dysfunction during pregnancy especially when a deficiency of iodine intake exists and when thyroid reserve is not sufficient.
\end{abstract}

Aim: To assess thyroid function tests during pregnancy.

Subjects and methods: cross-sectional study was carried out on 100 pregnant women attending obstetrics outpatient clinic in Suez Canal university hospitals were invited to enroll in the study. At end of study, the blood samples were assessed for free T3, free T4 and TSH.

Results: Our study revealed that most of pregnant women had normal thyroid functions (51\%), while subclinical hypothyroidism (39\%) was the most prevalent disorder followed by clinical hypothyroidism (6\%) and isolated hypothyroxinemia (4\%).

Conclusion: The most prevalent pattern of thyroid dysfunction in pregnant women was subclinical hypothyroidism.

Keywords: endocrine disorder, thyroid hormones, physiological state, pregnant women
Volume 5 Issue 6 - 2019

\section{Ahmed Abdelmonem Aboelroose \\ Faculty of medicine, Suez Canal University, Egypt}

Correspondence: Ahmed Abdelmonem Aboelroose, Lecturer of obstetrics and gynecology, Faculty of medicine, Suez Canal university, Egypt,Tel 0I22356234I, Email aaboelroos@gmail.com

Received: November 18, 2019 | Published: December 04, 2019
Abbreviations: ATA, American thyroid association; HCG human chorionic gonadotrophin; TSH, thyroid stimulating hormone.

\section{Introduction}

Thyroid dysfunction is the second most common endocrine disorder, only after diabetes mellitus, affecting females in reproductive age group. Thyroid gland performs it's very important functions. These hormones also play a very important role in maturation of fetal brain cells. ${ }^{1}$ Pregnancy is a physiological state in which significant changes in thyroid function occur. Several factors contribute to these changes. Indeed, since the beginning of pregnancy, the HCG (human chorionic gonadotrophin) secreted by the placenta, given its homology of structure with TSH (thyroid stimulating hormone), exerts a stimulatory effect on thyroid gland leading to an increase in the secretion of thyroid hormones (T3 and T4) and a decrease in TSH, especially during the first trimester. ${ }^{2}$ In addition, pregnancy induces increase in metabolic function and requires a higher production of thyroid hormone. Demand for iodine intake increases especially as there is a greater urinary excretion of iodine and a transfer of iodine to the fetus when his thyroid becomes functional. ${ }^{3}$

All these factors could contribute to thyroid dysfunction during pregnancy especially when a deficiency of iodine intake exists and when thyroid reserve is not sufficient

\section{Subject and methods}

\section{Study design}

The present study was descriptive cross-section study. The study was carried out in Suez Canal university hospitals, obstetrics outpatient clinic, Ismailia, Egypt.

\section{Study population}

A total number of one hundred pregnant women attending obstetrics outpatient clinic at Ismailia university hospitals for antenatal care were invited to enroll in this study. The included patients had the following criteria:

a. Age: 18 -40years old pregnant females.

b. Single embryo.

c. First, second and third trimester of pregnancy.

d. Patients were excluded if they were:

e. Smokers.

f. Patients on medications that affect thyroid profile as amiodarone.

g. Patients refuse to be included in the study.

h. Women with a family history of thyroid disease.

i. Women with a history of either miscarriage or preterm delivery.

j. Women with certain other autoimmune disorders which are frequently associated with autoimmune thyroid dysfunction as vitiligo, adrenal insufficiency etc.

$\mathrm{k}$. Women with prior therapeutic head or neck irradiation.

\section{Tools and data collection}

Patients were assessed through personal interview. The assessment included History taking, general and neck examination. All patients had thyroid function test included TSH, FT3,FT4.As no trimesterspecific reference ranges for the assessment of thyroid function in pregnancy had been established for the Egyptian population at the time of the study, the Guidelines of the American Thyroid Association (ATA) for the Diagnosis and Management of Thyroid Disease during Pregnancy and Postpartum recommendations were applied. ${ }^{4}$

Primary objective: to assess magnitude of thyroid dysfunction problem during pregnancy and to predict when pregnant woman needs thyroid disorder management. 
Secondary objective: to avoid pregnancy complications associated with either hypo or hyperthyroidism.

\section{Statistical analysis}

Collected data was processed using SPSS 18. Quantitative data were presented as mean $\pm \mathrm{SD}$ while qualitative data were expressed as numbers and percentages. Unpaired T-test was used to test the significance of difference between quantitative data while Chi-Square test was used for qualitative data. A probability value (P-value) less than 0.05 was considered significant. Results were presented in tables and graphs

\section{Results}

In the present study, we assessed various thyroid disorders among one hundred $(\mathrm{N}=100)$ pregnant women.

Table 1 demonstrates the demographic data of the studied population; it showed that study population age ranged from 18 to 39 years with average of $28.5 \pm 5$ years. Sixty percent of studied population was younger than thirty years. Chart 1 showed that $49 \%$ of the studied population had subclinical hypothyroidism was the most prevalent disorder representing 39\% of the total population. Table 2 showed that highest prevalence of hypothyroidism was in the second trimester $(50 \%)$ followed by first trimester $(48.9 \%)$ and finally third trimester $(47.6 \%)$ which showed that no statistically significant relationship between each trimester of pregnancy and development of thyroid disorders. Concerning the Relation between thyroid function and age. Table 3 showed that $3(50 \%)$ of patients with clinical hypothyroidism were less than 30 years and $3(50 \%)$ were $30-40$ years old and no statistically significant relationship between thyroid functions and age. Table 4 showed that according to ATA guide-lines 2017 the thyroid function laboratory results of the enrolled pregnant women were as $77 \%$ has a normal thyroid function, $13 \%$ diagnosed to have subclinical hypothyroidism, $3 \%$ clinical hypothyroidism and $7 \%$ isolated hypothyroxinemia. So, there is over estimation of thyroid dysfunction using ATA guide lines 2017 and under estimation using only selected high risk population screening

Table I demonstrates the demographic data of the studied population

\begin{tabular}{lll}
\hline $\begin{array}{l}\text { Demographic } \\
\text { characteristics }\end{array}$ & No. & $\%$ \\
\hline Age & & \\
$18-29$ & 60 & 60 \\
$\geq 30$ & 40 & 40 \\
Min.-Max. & $18.0-39.0$ & \\
Mean \pm SD & $28.53 \pm 4.90$ & \\
Median & 28 & \\
Residency & & 28 \\
Rural & 28 & 72 \\
Urban & 72 & \\
Gravidity & & 19 \\
Nullipara & 19 & 81 \\
Multigravida & 81 &
\end{tabular}

Table 2 relationship between every trimester and thyroid function

\begin{tabular}{|c|c|c|c|c|c|c|c|c|}
\hline \multirow[t]{4}{*}{ Thyroid function } & \multicolumn{6}{|c|}{ Trimester } & \multirow[t]{3}{*}{$x^{2}$} & \multirow[t]{3}{*}{$M C_{p}$} \\
\hline & Ist & & 2nd & & $3 r d$ & & & \\
\hline & $(n=45)$ & & $(n=3$ & & $(n=21)$ & & & \\
\hline & No. & $\%$ & No. & $\%$ & No. & $\%$ & & \\
\hline Normal & 23 & 51.1 & 17 & 50 & 11 & 52.4 & 0.848 & 1 \\
\hline Sub clinical hypothyroidism & 17 & 37.8 & 14 & 41.2 & 8 & 38.1 & & \\
\hline Clinical hypothyroidism & 3 & 6.7 & 2 & 5.9 & 1 & 4.8 & & \\
\hline Isolated hypothyroxinemia & 2 & 4.4 & I & 2.9 & 1 & 4.8 & & \\
\hline
\end{tabular}

Table 3 Relation between thyroid function and age (years) $(n=100)$

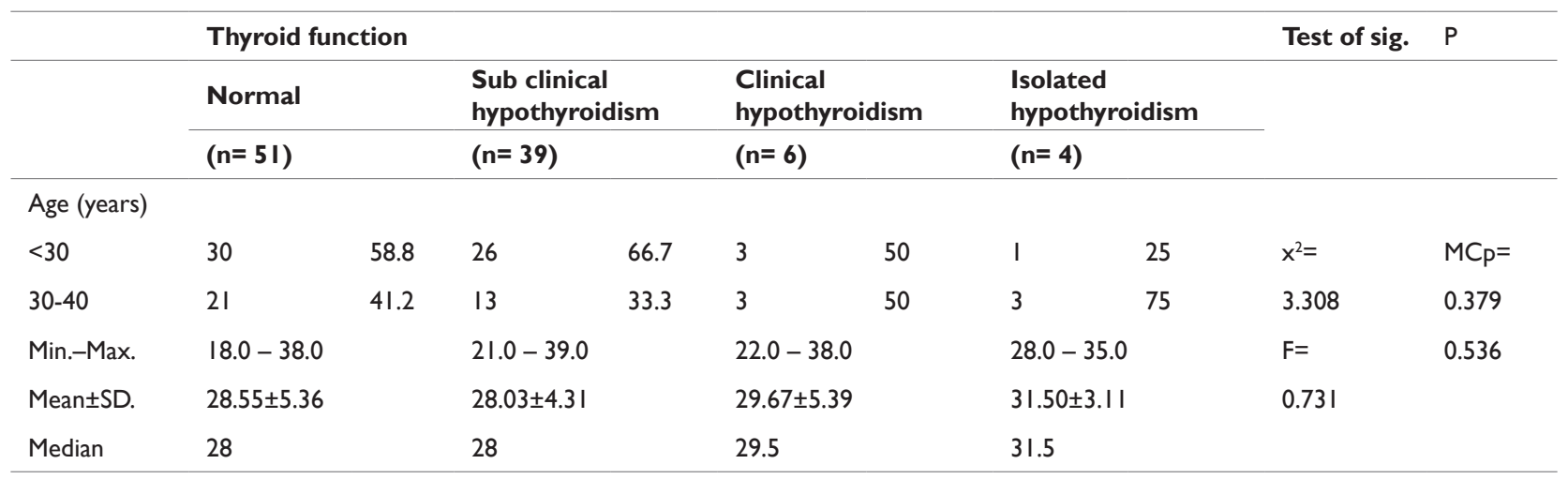


Table 4 Prevalence of thyroid dysfunction according to ATA guide-lines 2017 $(n=100)$

\begin{tabular}{lll}
\hline & No. & $\%$ \\
\hline Normal & 77 & 77 \\
Subclinical hypothyroidism & 13 & 13 \\
Clinical hypothyroidism & 3 & 3 \\
Isolated hypothyroxinemia & 7 & 7 \\
\hline
\end{tabular}

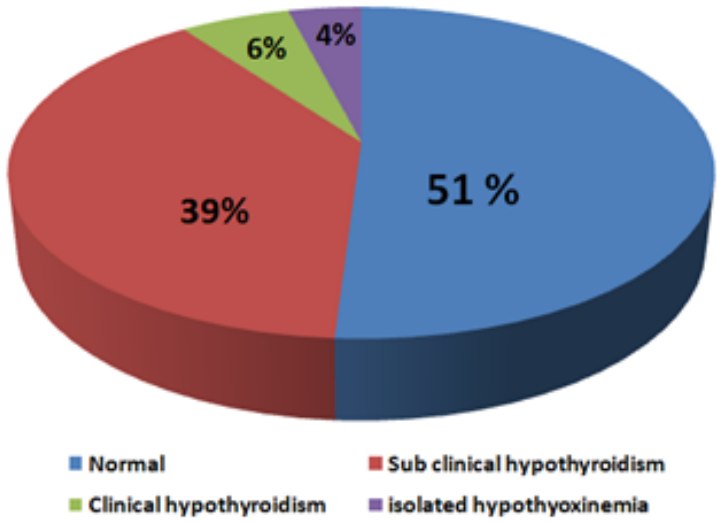

Chart I Percentage of thyroid dysfunction.

\section{Discussion}

Considerable changes occur in the thyroid hormone physiology and thyroid gland anatomy during pregnancy. Specifically, the thyroid gland of the pregnant woman is characterized by glandular hyperplasia, increased vascularity, and an approximately 30\% increased volume, despite a normal echo structure. ${ }^{5}$ Furthermore, thyroid function test changes can occur because of androgen-mediated increases in thyroid-binding globulin and a decline in iodide because of increased renal clearance and placental loss. ${ }^{6}$ In the present study, we assessed various thyroid disorders among one hundred $(n=100)$ pregnant women attending obstetric outpatient clinic in Suez Canal university hospitals. These pregnant women ages' ranged from 18 to 39 years with average of $28.5 \pm 5$ years. It was found that sixty percent of studied population was younger than thirty years old. Seventy two women were residents in urban areas. Most women were multigravida, representing eighty one percent. Majority of women were in their first trimester $(45 \%)$ followed by second trimester (34\%), while least cases were in third trimester.

Our study found that most of pregnant women $(n=51)$ had normal thyroid functions, while subclinical hypothyroidism $(n=39)$ was the most prevalent disorder followed by clinical hypothyroidism $(n=6)$ and isolated hypothyroxinemia $(\mathrm{n}=4)$.

In Egypt, across-sectional study among 168 pregnant women who attend the outpatient obstetric clinic at Ain Shams university hospital, they were subdivided into high-and low-risk groups for thyroid disease according to ATA guidelines as well as groups by trimester and shows that prevalence of hypothyroidism was $56 \%$ in the high risk group and $44.6 \%$ in the-low risk group with no statistically significant differences.?

According to our findings, the overall incidence of thyroid disorders in low risk pregnant women was $49 \%$.Our study came in accordance with who noted hypothyroidism in $55.8 \%$ of pregnant women in a study held in Egypt mostly subclinical hypothyroidism (42.3\%). A study conducted in Jordan on 322 pregnant women in the first trimester where $20.8 \%$ of women were considered to have subclinical hypothyroidism comparable to $37.8 \%$ in this study. ${ }^{8}$ Across-sectional study in referral hospital in Spain among 2509 pregnant women in the first trimester reported prevalence of thyroid dysfunction at $16 \%{ }^{9}$

In china, a multicenter cohort study among 2899 pregnant women enrolled during their first trimester of gestation demonstrated a prevalence of thyroid dysfunction at $10.2 \%$. This study did not use the trimester specific reference ranges recommended by American Thyroid Association. ${ }^{10}$ A prospective observational study in India among 1000 pregnant women attending a tertiary public hospital in the first trimester reported a prevalence of thyroid dysfunction at $14.3 \%$, with subclinical hypothyroidism being the most common. ${ }^{11}$ In Sudan, cross-sectional hospital-based study among 500 pregnant Sudanese women aged 15-45 years in all trimesters, found a prevalence of $9.4 \%$ this study use national reference ranges instead of the ATA trimester specific reference ranges. ${ }^{12} \mathrm{~A}$ cross-section study carried out in Tunisia among 1519 pregnant women in all trimesters demonstrated prevalence of thyroid dysfunction at $9.7 \% .{ }^{13}$ In Saudi Arabia, acrosssectional study among 384 pregnant women the majority $(89.3 \%)$ of women aged less than 40 years and obesity was found to be $53.9 \%$, the mean age was 32.6 , the prevalence of subclinical hypothyroidism was $13 \%$, and Chi-square analysis indicated that age and obesity were not significantly associated with subclinical hypothyroidism which is consistent with our results. ${ }^{14}$ This variability in thyroid disorders prevalence in pregnant women could be explained by the iodine status of study population, the sample size, the references used to define thyroid status or by ethnicity specificity.

Another study in Africa comparing the prevalence of thyroid functions in pregnancy using 2011ATA guidelines and 2017 ATA guidelines. In a study by Awede et al., ${ }^{15}$ Two hundred and forty (240) pregnant women were included in the study. It showed that Thyroid dysfunction was present in $24.17 \%$ of the pregnant women. Hypothyroidism was observed in $22.5 \%$ of subjects with $19.17 \%$ of subclinical hypothyroidism, $0.87 \%$ of overt hypothyroidism and $2.5 \%$ of hypothyroxinemia while hyperthyroidism was present in $1.67 \%$ of the subjects, while using 2017 ATA guidelines (TSH upper reference limit of $4.0 \mathrm{mU} / \mathrm{L}$ ). In this case, thyroid disorders were present in $14.58 \%$ of pregnant women. The frequency of subclinical hypothyroidism, overt hypothyroidism, hypothyroxinemia and hyperthyroidism were respectively $9.58 \%, 0.42 \%, 2.91 \%$ and $1.67 \%$.

In our study we did similar comparison and we found that prevalence of thyroid dysfunction according to 2011 ATA reference ranges was $49 \%$ (39\% $\mathrm{SCH}, 6 \% \mathrm{OH}, 4 \%$ isolated hypothyroxinemia), while according to 2017 ATA reference ranges was $23 \%(13 \% \mathrm{SCH}$, $3 \% \mathrm{OH}$ and $7 \%$ isolated hypothyroxinemia).

It was found that there was no statistically significant relationship between trimester of pregnancy and development of thyroid disorders. These results support a study by Brent who recommended performing universal screening for thyroid dysfunction early in pregnancy as well as during the second- and third trimesters. ${ }^{16}$ Moleti found that $40 \%$ of screened women were diagnosed with hypothyroidism in the early and late second trimester and would not have been identified, if we had limited our observation to the first thyroid function test alone. ${ }^{17}$ Several researchers have also expressed great concern regarding hypothyroidism during the first trimester, as the fetus at that period is 
entirely dependent on maternal thyroid hormone levels, having not yet become able to make its own endogenous supply. ${ }^{18}$

Among different clinical parameters presented at clinics, it was found that theses parameters didn't show any statistically significant relationship with certain thyroid disorders. This observation could be explained by similarity of pregnancy associated physiological changes and signs of hypo and hyperthyroidism.

Most of women were overweight $(n=62)$, this could be related to the common belief among Egyptian pregnant women that increased caloric intake during pregnancy is encouraged for healthier fetal development. Although the weight gain in pregnancy did not correlate with hypothyroidism, it sheds lights on the importance of increasing awareness about adequate weight gain during pregnancy.

However, women with normal body mass index came next $(n=25)$ and least cases were obese $(n=13)$. Also there was no statistically significant relationship between thyroid disorders and body mass index of pregnant women. These results are in contrast with Dima et al with a study was done on 920 pregnant females in Lebanon found that women with a BMI $>35 \mathrm{~kg} / \mathrm{m}^{2}$ might have a greater risk of developing hypothyroidism, this may be due to that maximum BMI in our study was $33 \mathrm{~kg} / \mathrm{m} 2$ and also difference in sample size. They found that prevalence of hypothyroid disorders with pregnancy was $17.1 \%$ which is lower than our finding (49\%). ${ }^{19}$

In the current study, we had found that there was no statistically significant relationship between development of thyroid disorders and age of women, nor gravidity status nor the trimester. This comes in contrary with Awede et al., ${ }^{15}$ who observed positive relationship between hypothyroidism with age, trimester and number of gravidity. This may be due to the difference in the sample size, population characteristics.

\section{Conclusion}

In conclusion, most of the studies that were concerned about Assessment of Thyroid Function in Pregnant Females noted that there was no statistically significant relationship between development of thyroid disorders and age of women, nor gravidity status .Our current study was in agreement with most of these studies. Only few studies were disagreed with these findings. Also we concluded that these findings from the present study underscore the prevalence of hypothyroidism among pregnant women and the most prevalent pattern of thyroid dysfunction in pregnant women was subclinical hypothyroidism. Factors affecting thyroid dysfunction during pregnancy especially subclinical hypothyroidism needs further studies to be assessed.

\section{Acknowledgments}

None.

\section{Conflicts of interest}

No conflicts to declare.

\section{Funding}

None.

\section{References}

1. Jansen J, Friesemae C, Milici C, et al. Thyroid hormone trans-porters in health and disease. Thyroid. 2005;15(8):757-768.
2. Lazarus I, Brown RS, Daumerie C, et al. European thyroid association guidelines for the management of subclinical hypothyroidism in pregnancy and in children. Eur Thyroid J. 2014;3(2):76-94.

3. Lazarus JH. Thyroid Function in Pregnancy. 2010;97:137-148.

4. Stagnaro-Green A, Abalovich M, Alexander E, et al. American Thyroid Association Taskforce on Thyroid Disease During Pregnancy and Postpartum. Guidelines of the American Thyroid Association for the diagnosis and management of thyroid disease during pregnancy and postpartum. Thyroid. 2011;21(10):1081-125.

5. American College of Obstetricians and Gynecologists. Practice bulletin no. 148: thyroid disease in pregnancy. Obstet Gynecol. 2015;125(4):9961005 .

6. Casey BM, leveno KJ. Thyroid disease in pregnancy. Obstetric Gynecology. 2006;108:1283-1292.

7. Ahmed IZ, Eid YM, El-Orabi H, et al. Comparison of universal and targeted screening for thyroid dysfunction in pregnant Egyptian women. Eur J Endocrinol. 2014;171:285-291.

8. Alkafajei A, Amarin Z, Alazaizeh W, et al. Prevalence and risk factors for hypothyroidism in Jordanian women: comparison between different reference ranges. East Mediterr Health J. 2012;18(2):132-136.

9. Dieguez M, Herrero A, Avello N. Prevalence of Thyroid Dysfunction in Women in Early Pregnancy. Clin Endocrinol (Oxf). 2016;84(1):121126.

10. Wang W, Teng W, Shan Z. The prevalence of thyroid disorders during early pregnancy in china: The benefits of universal screening in the first trimester of pregnancy. Eur J Endocrinol. 2011;64:263-268.

11. Dhanwal DK, Prasad S, Agarwal AK. High Prevalence of subclinical hypothyroidism during first trimester of pregnancy in North India. $J$ Endocr Metab. 2013,17:281-284.

12. Saeed AK, Yassin K, Elmahdi EMA, et al. The Prevalence of Thyroid Dysfunction among Sudanese Pregnant Women. SOJ Gynecol Obstet Womens Health. 2015;1(1):5.

13. Feki M, Omar S, Menif O. Thyroid disorders in pregnancy. Frequency and association with selected diseases and obstetrical complications in Tunisian women. Clin Biochem. 2008;41(12):927-931.

14. Al-Shanqeeti SA, Al-khudairy YN, Alabdulwahed AA, et al. Prevalence of subclinical hypothyroidism in pregnancy in Saudi Arabia. Saudi Med J. 2018;39:254-256.

15. Awede B, Hounnou M, Tshabu-Aguemon C, et al. Thyroid Function in Pregnant Women from a West-African Population. Open Journal of Molecular and Integrative Physiology. 2018;8:1-11.

16. Brent GA. Diagnosing thyroid dysfunction in pregnant women: is case finding enough? Journal of Clinical Endocrinology and Metabolism. 2007;92:39-41.

17. Moleti M, Lo Presti VP, Mattina F, et al. Gestational thyroid function abnormalities in conditions of mild iodine deficiency: early screening versus continuous monitoring of maternal thyroid status. European Journal of Endocrinology. 2009;160:611-617.

18. Springer D, Zima T, Limanova Z. Reference intervals in evaluation of maternal thyroid function during the first trimester of pregnancy. European Journal of Endocrinology. 2009;160(5):791-797.

19. Ezzeddine D, Hamadi C, Abbas HA, et al. Prevalence and Correlation of Hypothyroidism With Pregnancy Outcomes Among Lebanese Women. Journal of the Endocrine Society. 2017;1(5):415-422. 